\title{
Captures
}

Figures, théories et pratiques de l'imaginaire

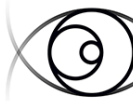

C A P T U R E S

\section{Être un artiste contemporain et être autochtone} Quelques réflexions sur la polémique autour de Jimmie

\section{Durham}

\section{Jean-Philippe Uzel}

Volume 3, Number 1, May 2018

La notion d'«autochtonie»

URI: https://id.erudit.org/iderudit/1055839ar

DOI: https://doi.org/10.7202/1055839ar

See table of contents

Publisher(s)

Figura, Centre de recherche sur le texte et l'imaginaire

ISSN

2371-1930 (digital)

Explore this journal

Cite this article

Uzel, J.-P. (2018). Être un artiste contemporain et être autochtone : quelques réflexions sur la polémique autour de Jimmie Durham. Captures, 3(1).

https://doi.org/10.7202/1055839ar
Article abstract

This article focuses on the controversy, which came out in June 2017, about the Cherokee identity of the well-known contemporary artist Jimmie Durham. Cherokee artists, intellectuals, and activists published an open letter

denouncing Durham's Cherokee identity as a fraud. This article does not intend to settle this complex debate, but rather wishes to examine how this argument shed some light on the notion of "Indigeneity" in the arts.

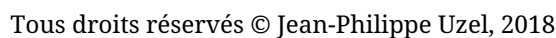

\section{(c) (i) $\Theta$}

This document is protected by copyright law. Use of the services of Érudit (including reproduction) is subject to its terms and conditions, which can be viewed online.

https://apropos.erudit.org/en/users/policy-on-use/ 


\section{Être un artiste contemporain et être autochtone}

\section{Quelques réflexions sur la polémique autour de Jimmie Durham Jean-Philippe Uzel}

Résumé :

Cet article revient sur la polémique qui a éclaté en juin 2017, lorsque des artistes, des intellectuels et des militants cherokees ont publié une lettre ouverte dénonçant comme frauduleuse l'identité cherokee de l'artiste Jimmie Durham, l'une des figures les plus reconnues de l'art contemporain autochtone. Loin de vouloir trancher un débat aux ramifications complexes, cet article entend plutôt restituer les termes de la querelle et examiner la façon dont cette controverse éclaire la notion d'« autochtonie ».

This article focuses on the controversy, which came out in June 2017, about the Cherokee identity of the wellknown contemporary artist Jimmie Durham. Cherokee artists, intellectuals, and activists published an open letter denouncing Durham's Cherokee identity as a fraud. This article does not intend to settle this complex debate, but rather wishes to examine how this argument shed some light on the notion of "Indigeneity" in the arts.

Incredible as it may seem, Durham was not an Indian...

Jimmie Durham au sujet de Douglas Durham, chef de la sécurité de l'American Indian Movement1

Jimmie Durham (1940-) est aujourd'hui l'artiste contemporain autochtone le plus connu dans le monde de l'art occidental. Le Musée d'art moderne de la Ville de Paris en 2009 et le Musée d'art contemporain d'Anvers en 2012 lui ont déjà consacré des rétrospectives couvrant quarante ans de création. Depuis son installation en Europe, il y a vingt-cinq ans, son travail est inclus dans tous les grands rendez-vous internationaux de l'art contemporain (la Documenta de Kassel, la Biennale de Venise...), mais aussi dans les grandes expositions autochtones organisées, entre autres, au Canada (Terre Esprit Pouvoir à Ottawa en 1992, Close Encounters à Winnipeg en 2011, Sakahàn à Ottawa en 2013). Son engagement au sein de l'American Indian Movement (AIM) dans les années 70, entre autres comme directeur de l'International Indian Treaty Council (IITC) à l'ONU, organisme qui allait semer les germes de ce qui deviendra en 2007 la Déclaration des Nations Unies sur les droits des peuples autochtones, a fait de lui une voix respectée sur la scène internationale. Ajoutons que Jimmie Durham est certainement l'une des personnes ayant le plus réfléchi et écrit sur ce que signifie être un « artiste contemporain autochtone ", et que ses articles ont déjà fait l'objet de deux imposants recueils (Durham 1993, 2014). On comprendra pourquoi la polémique qui a éclaté en juin 2017 , 
lorsque des artistes, des intellectuels et des militants cherokees ont publié une lettre ouverte dénonçant comme frauduleuse l'identité cherokee de l'artiste, a eu autant de retentissement $\underline{\text { }}$.

Précisons d'emblée que le présent article, écrit par un non-Autochtone, n'a pas la prétention de trancher un débat aux ramifications complexes. Celui-ci oppose deux camps. D'un côté, des militants et artistes cherokees qui présentent Jimmie Durham comme un usurpateur ayant construit toute sa carrière, artistique et politique, sur un mensonge : celui d'être un Cherokee non enregistré. De l'autre, les défenseurs de l'artiste qui affirment que ses dénonciateurs sont seulement motivés par le ressentiment devant sa carrière éblouissante et utilisent l'argument du «blood quantum 3 » pour en finir avec quelqu'un qui n'a cessé de critiquer la légitimité des trois tribus (ou « bandes ») qui représentent officiellement les Cherokees aux États-Unis et leur conception réductrice, voire raciste, de l'identité indienne 4 . Ces questions dépassent largement la carrière de Jimmie Durham et engagent la politique coloniale des États-Unis au $\mathrm{XIX} \mathrm{X}^{\mathrm{e}}$ siècle, qui eut pour résultat la spoliation quasi-complète des terres autochtones. Nous ne cherchons pas ici à résoudre ces querelles historiques, mais aimerions plutôt restituer les termes de la dispute et mettre l'accent sur un des grands constats qui en ressortent : l'incompréhension persistante qui existe encore aujourd'hui entre un monde de l'art contemporain globalisé et des communautés autochtones qui luttent pour leur survie culturelle. Au-delà du cas Jimmie Durham, ce débat permet ainsi, croyons-nous, de jeter quelques lumières sur la notion d'« autochtonie » dans les arts contemporains.

\section{L'acte d'accusation : une imposture de près de 50 ans}

Ce devait être le grand retour de Jimmie Durham en Amérique après trente ans d'absence. La conservatrice du Hammer Museum de Los Angeles, Anne Ellegood, avait convaincu l'artiste, après des années de démarches, d'organiser une grande rétrospective de son travail à travers les États-Unis et le Canada $\underline{5}$, Jimmie Durham. At the Center of the World (2017-2018). En mars 2017, quelques semaines après l'ouverture sans heurts de l'exposition à Los Angeles, Jori Finkel dans le New York Times consacrait un article au retour de l'enfant prodige, en résumant les grandes étapes d'une carrière bien remplie. À cette occasion, l'artiste, interrogé depuis Naples (Italie), où il réside, son état de santé ne lui permettant pas de se déplacer aux États-Unis, déclarait : «I am perfectly willing to be called Cherokee [...]. But I'm not a Cherokee artist or Indian artist » (Finkel, 2017). Cette protestation de Jimmie Durham allait être brandie quelques mois plus tard par ses adversaires comme un aveu de culpabilité - et même faire l'objet d'une intervention artistique de l'artiste Sioux-Dakota David Bernie. Elle ne faisait pourtant que réitérer la position défendue par l'artiste depuis trente ans. Durham s'est en effet toujours battu pour que sa pratique artistique ne soit pas enfermée dans une catégorie identitaire réductrice, celle de « l'art indien ». Notons que cette posture, qui consiste à se définir non pas comme un artiste autochtone, mais plutôt comme un artiste qui est aussi un Autochtone, est aujourd'hui 
largement répandue, du moins au Canada (Hessel, 2010: 189).

L'article du New York Times mentionnait également la loi fédérale Indian Arts \& Crafts Act (IACA) de 1990, qui a motivé la décision de Jimmie Durham, qui vivait au Mexique depuis 1987, de s'établir définitivement en Europe à partir de 1994. Cette loi, votée pour lutter contre l'appropriation culturelle, exige que les artistes produisant de l'art dit « indien » prouvent leur affiliation à l'une des 565 tribus vivant sur le territoire des ÉtatsUnis. En un mot, ils doivent être des artistes dûment enregistrés (enrolled) par l'État fédéral. Jimmie Durham s'étant toujours présenté comme un Cherokee non enregistré (unenrolled), c'est-à-dire n'étant reconnu par aucune des trois tribus cherokees officielles, n'avait désormais plus le droit de vendre son art aux États-Unis, sous peine d'emprisonnement. Le vote de l'IACA a créé un virulent débat dans le monde de l'art contemporain autochtone aux États-Unis et à l'étranger. Plusieurs artistes, dont Durham, ont qualifié cette loi de raciste, car elle exigeait de faire la démonstration d'un pourcentage de sang (blood quantum) pour obtenir le droit d'exercer son art (Turney, 1999). L'artiste Blackfoot Joane Cardinal-Schubert a par exemple consacré une œuvre, Preservation of a Species. DECONSTRUCTIVISTS (This is the house that Joe built)(1990), à la dénonciation de cette loi jugée inique. On pouvait y lire l'inscription manuscrite suivante : " you don't get $50 \%$ or $25 \%$ or $16 \%$ treatment when you experience racism - It is always $100 \% »$. On comprend que Durham ait été très aigri par l'adoption d'une loi qui venait mettre un terme à sa carrière artistique aux États-Unis, et qui entérinait également l'échec de son engagement politique pour les droits civiques dans les années 70 : « it is a sign of how my side, how badly the American Indian Movement side lost 》 (Durham cité par Papastergiadis, 1996: 31). Durham a publié plusieurs textes caustiques sur les conséquences de l'IACA qui ont souvent été interprétés au premier degré par ses adversaires. Par exemple, en réponse à un article très élogieux que l'historienne de l'art Lucy Lippard lui avait consacré dans les pages de la revue Art in America en 1993, où elle évoquait les origines cherokee de l'artiste (Lippard, 1993), Durham a jugé approprié d'envoyer une lettre aux éditeurs de la revue pour remercier l'historienne de l'art de ses propos généreux et d'ajouter sur un ton amer: «I am not Cherokee. I am not an American Indian. This is in concurrence with recent US legislation, because I am not enrolled on any reservation or in any American Indian community. » (Durham, 2017 [1993]). Certains commentateurs prirent à l'époque cette déclaration au pied de la lettre : « Durham apparently took the threat seriously. He has since written Art in America citing the law and declaring, "I am not an American Indian." (Tilove, 1993.) Cette phrase de Durham, tout comme sa déclaration dans le New York Times citée plus haut, est elle aussi abondamment reprise dans la controverse actuelle. Elle apparaît par exemple citée plusieurs fois dans le numéro d'automne du First American Art Magazine, entièrement à charge contre l'artiste (Meredith, 2017).

En accordant le statut d'« artiste indien » aux seuls artistes enregistrés, l'IACA ravivait les violentes querelles qui divisèrent les Premières Nations tout au long du XIXe siècle et dans la première moitié du $X X^{e}$ au sujet de 
la reconnaissance ou non des lois coloniales. Une de ces premières lois fut l'Indian Removal Act de 1830, qui rendait légale la déportation des « cinq tribus civilisées » (Five Civilized Tribes) à l'ouest du Mississipi afin de redistribuer leurs terres aux colons européens (Woodward, 1963: 192-218). Les Cherokees, une des cinq tribus, furent ainsi déplacés de force en 1838 de leurs terres ancestrales situées dans les États du Tennessee, de la Géorgie, de la Caroline du Nord et du Sud vers le Territoire indien (Indian Territory트) — cette déportation de plusieurs milliers de kilomètres, qui entraîna d'énormes pertes humaines, est connue sous le nom de « route des larmes ». La stratégie de dépossession de l'État fédéral fut poursuivie par le Dawes Act de 1887 (aussi connu comme l'Allotment Act), qui attribuait aux Cherokees enregistrés, et seulement à ceux-ci, des parcelles de terre et leur offrait également la citoyenneté américaine. Cette loi, dont l'objectif déclaré consistait à assimiler les membres des Premières Nations dans la société américaine et de s'approprier les terres du Territoire Indien, qui allait devenir en 1907 l'État de l'Oklahoma, déclencha une lutte fratricide entre les Cherokees qui acceptèrent les termes de la loi et ceux qui s'y opposèrent. Les ancêtres de Jimmie Durham appartenaient, selon lui, à cette dernière catégorie; et c'est pour cette raison qu'ils ne furent jamais reconnus par les États-Unis (Papastergiadis, 1996: 31-33). Jimmie Durham a consacré plusieurs textes à la « guerre civile » qui a déchiré la Nation Cherokee des années 1880 aux années 1920 (Durham, 1993: 126135; 2014: 275-284) et affirme que l'IACA n'est que le prolongement de ce conflit dans le domaine de l'art. On comprend bien que son désaccord ne se limitait pas à son droit de vendre ou de ne pas vendre son art sur le sol américain. C'est également pour protester contre ce qu'il appelle la politique « d'apartheid » des États-Unis que Jimmie Durham a décidé de quitter définitivement son pays natal et de vivre en exil. Il a toutefois accepté que sa rétrospective Jimmie Durham. At the Center of the Worldcircule aux USA - ce qui peut surprendre lorsque l'on connaît ses prises de position très tranchées contre son pays d'origine -, l'IACA ne s'appliquant pas à cette exposition puisque les œuvres n'y sont pas à vendre.

II nous a semblé important de revenir sur les termes de la polémique entourant le vote de l'IACA car, contrairement à ce que prétendent les défenseurs de l'artiste, les termes de la querelle qui a débuté en juin 2017 sont fort différents de ce qu'ils étaient en 1990. À l'époque le débat portait sur le fait qu'un membre d'une Première Nation non enregistré n'était pas reconnu par l'État fédéral en tant que véritable Autochtone. Or, le débat actuel porte ni plus ni moins sur la « fraude ethnique » sur laquelle Durham aurait construit l'ensemble de sa carrière. II ne s'agit plus de savoir si un Cherokee unenrolled est oui ou non un Cherokee, mais bien de savoir si Jimmie Durham est un imposteur. Les arguments des signataires de la lettre d'Indian Country Today, s'appuyant sur une enquête généalogique poussée 7 , affirment que Durham n'a aucun ancêtre cherokee et que ses origines autochtones sont pure affabulation. Partant de la date de naissance de l'artiste, le 10 juillet 1940, ils affirment que celui-ci n'est pas né, comme il le prétend, à Washington en Arkansas mais à Harris au Texas d'une famille blanche sans aucun lien avec la Nation Cherokee. Ils en veulent pour preuve que les parents, les 
grands-parents et même l'arrière-grand-mère de Jimmie Durham apparaissent dans les recensements fédéraux de la population américaine et à aucun moment dans les recensements propres à la Nation Cherokee. Or, celle-ci est une des Premières Nations qui ont été le plus recensées, même avant la grande déportation de 1838. Les ancêtres de Durham, lesquels auraient migré à l'ouest entre 1817 et 1835 (Ellegood, 2017a: 25), et appartiendraient donc à la catégorie des Early Settlers, devraient en toute logique apparaître dans ces premiers recensements. Ce n'est pas le cas. L'argument semble implacable et a d'autant plus de poids que Durham n'a pas daigné y répondre, s'en tenant à la position qu'il avait énoncée dans l'entretien du New York Times en mars 2017, à savoir qu'il n'avait pas de réponse à apporter aux personnes qui le critiquaient au sujet de son identité (Finkel, 2017)으. Notons au passage que la Nation Cherokee a été victime ces dernières années de nombreuses impostures ethniques (Russell, 2008) et que les Cherokees autoproclamés sont, selon le recensement fédéral de 2010, plus nombreux que la totalité des citoyens reconnus par les trois tribus cherokees - phénomène qui rappelle celui des Métis au Canada, où certaines provinces comme le Québec ont connu une augmentation de $149 \%$ de la population métisse entre 2006 et 2016.

Les signataires de la lettre d'Indian Country Todays'en prenaient également, comme le prouve le titre de leur lettre « Dear Unsuspecting Public, Jimmie Durham Is a Trickster », à la naïveté du public occidental et plus particulièrement à tous ces historiens de l'art et commissaires qui écrivent sur Jimmie Durham et l'exposent depuis des années sans avoir jamais pris la peine de vérifier la véracité de ses affirmations, qu'il s'agisse de ses origines ou de l'usage qu'il fait de la langue et des concepts cherokees. Cet aveuglement du monde de l'art contemporain face aux affabulations présumées de Durham traduirait, selon eux, tant la persistance des idées reçues sur les « Indiens » - Jimmie Durham incarnant à la perfection l'image de l'Indien renégat dans l'imaginaire occidental - , que le peu de volonté de ce même milieu de se rapprocher des communautés autochtones. Reconnaissons que cet argument n'est pas nouveau. L'historienne de l'art et commissaire apache Nancy Marie Mitchell (maintenant Miltho), l'avait déjà utilisé en 1993 pour répondre à l'article de Lucy Lippard dans Art in America en insistant sur le fait que Durham répondait parfaitement aux attentes du public occidental en matière d'autochtonie : "However, your readers should be aware that this artist's fame stems from your ignorance. He knows your language, which boxes you need to check, which names to drop, and what injustices to cry » (Durham, 2017 [1993]).

Fait aggravant pour les contempteurs de Jimmie Durham : non seulement celui-ci est, selon eux, un fraudeur, mais son imposture a fonctionné au-delà de toute attente, puisqu'il est devenu au cours des années la "voix » d'autorité des Cherokees dans le monde de l'art. Pour étayer leur propos, ils ont lancé la requête « artiste cheerokee » sur la base de données JStor et obtenu 81 résultats répondant au nom de Jimmie Durham, alors que l'artiste suivant, Kay WalkingStick, n'en obtient que 18. C'est pour dénoncer l'énormité de cette imposture 
et le caractère frauduleux de l'exposition Jimmie Durham. At the Center of the Worldqu'ils ont décidé de prendre publiquement la parole. Leur lettre (Watts, 2017) a été suivie d'un grand nombre de prises de position provenant de chacun des deux camps $\stackrel{9}{\text {. }}$

\section{La défense : la plus longue performance de l'histoire de l'art contemporain}

Face à la gravité des accusations, la réaction en faveur de Jimmie Durham a été étrangement timorée, traduisant un véritable malaise de la part des soutiens de l'artiste. II faut dire que ceux-ci ont été gênés dans leur réplique par un événement inopiné. Le Walker Art Center de Minneapolis, lequel a accueilli l'exposition à partir du 22 juin 2017, était empêtré depuis quelques semaines dans une polémique impliquant la communauté autochtone des Dakotas. En effet, au mois de mai 2017 devait rouvrir le Minneapolis Sculpture Garden, une des composantes du Walker Art Center, après l'installation de dix-sept nouvelles sculptures publiques d'artistes contemporains. Parmi elles, Scaffold (2012), de l'artiste américain Sam Durant. Cette sculpture monumentale, qui se voulait une réflexion sur la peine de mort, se présentait comme un immense échafaud inspiré des véritables potences qui ont traversé l'histoire américaine : celles de Saddam Hussein, de John Brown..., mais également celles des trente-huit Dakotas qui furent pendus à Mankota (Minnesota) en 1862, à la fin de la guerre entre les États-Unis et les Sioux. Les membres de la communauté Dakota du Minnesota, lesquels n'avaient pas été consultés au sujet de l'œuvre de Sam Durant, exprimèrent publiquement leur colère face à une œuvre qui non seulement réveillait un passé douloureux, mais qui manquait également de respect aux victimes de la pendaison. Les responsables du Walker, avec l'accord de Sam Durant, réagirent rapidement en décidant de retirer l'œuvre. Cependant, le mal était fait : la polémique mettait en exergue le peu de sensibilité et de respect du monde de l'art contemporain face aux traumatismes des peuples autochtones. Et de nombreux articles de presse firent le lien entre Sam Durant et Jimmie Durham (Eldred, 2017), rappelant au passage qu'Anne Ellegood, la commissaire de Jimmie Durham. At the Center of the World avait envisagé dans les années de 2000 de réunir les deux artistes comme représentants officiels des États-Unis à la Biennale de Venise (Finkel, 2017)...

La défense la plus sérieuse de Jimmie Durham est venue, selon nous, de la part du Comanche Paul Chaat Smith, auteur du célèbre essai Everything You Know about Indians Is Wrong(2009) et un des plus anciens compagnons de route de Jimmie Durham, puisqu'il a dirigé le Treaty Council News dans les années 70, au moment où Durham était le directeur de l'IITC. Smith, invité par le Walker Art Center à prononcer une conférence le 31 août 2017 sur sa future exposition Americans au National Museum of the American Indian à Washington, consacra la première moitié de son intervention à la défense de son ami de longue date. II insista d'abord sur le fait qu'il connaissait Jimmie Durham depuis plus de quatre décennies et que la simple idée qu'il soit « blanc » lui semblait totalement saugrenue : 
If Jimmie Durham is a fraud, it would rank somewhere between two poles for me. The first would be finding out my parents were actually KGB officers. The second would be a colleague that you've known for decades, whose house you visited, who shared stories of their childhood and their siblings, you met their spouse and have friends in common, and you find one day every single thing they told you about their past was a lie (Smith, 2017).

Mais par-delà ses liens d'amitié, Smith isolait un point faible dans l'argumentation des auteurs de la lettre d'Indian Country Today, celui de s'en prendre uniquement au public occidental tenu responsable de la supposée fraude de Durham. II rappelait qu'avant de concerner le public occidental « naïf », l'imposture de Durham a d'abord été entérinée par les membres des Premières Nations. À commencer par les leaders de l'AIM et les centaines de Nations qui le choisirent pour porte-parole lorsqu'il fut nommé directeur de l'IITC dans les années 70. Ensuite, il est vrai que Jimmie Durham a été soutenu au cours de sa carrière par des critiques et commissaires occidentaux (Jean Fisher, Lucy Lippard, Guy Brett...), mais aussi par un nombre incalculable de commissaires, de critiques et d'artistes autochtones (Jaune Quick-to-See Smith, Jolene Rickard, Edgar Heap of Birds, Robert Houle, Richard W. Hill, Candice Hopkins, Greg Hill... et bien sûr Paul Chaat Smith). On peut faire le pari que ceux-ci se seraient sentis pour le moins mal à l'aise face à l'éventualité de collaborer avec un imposteur... En somme, Paul Chaat Smith mettait en évidence le fait que la question de l'identité de Jimmie Durham n'opposait pas seulement les artistes autochtones d'un côté et le monde de l'art occidental de l'autre, mais divisait aussi profondément la communauté artistique autochtone elle-même.

Parmi les défenseurs non-autochtones de Durham, la première personne à réagir à la lettre collective a été la commissaire de l'exposition, Anne Ellegood (2017b). Après avoir reconnu le droit des Premières Nations à décider elles-mêmes qui peut, ou non, prétendre au statut autochtone, la ligne de défense de la commissaire, visiblement embarrassée, était assez molle. Insistant sur l'aspect stérile de la querelle, elle soulignait que la lettre de l'Indian Country Today ne faisait que reprendre, sous un nouveau jour, les arguments entourant l'adoption de l'IACA de 1990 et que ces débats, de toute façon, étaient d'une grande complexité. Cet argumentaire a été repris en bloc par les responsables muséaux présentant l'exposition de Durham. Par exemple, le directeur du Withney Museum, Adam D. Weinberg, a publié une déclaration lorsque l'exposition est arrivée à New York dans laquelle on peut lire : "When this exhibition was previously presented in Los Angeles and Minneapolis, it revived longstanding debates about the artist's identification as Cherokee. Durham does not belong to any of the three Cherokee tribes and vigorously opposes the system of tribal enrollment on political grounds. » (Weinberg, 2018.) En revanche, un argument beaucoup plus étonnant, mais en même temps extrêmement révélateur de la piètre connaissance que les acteurs occidentaux possèdent des réalités autochtones, a été formulé dans un article du New York Times par le critique Holland Cotter, qui pose l'hypothèse que si l'artiste, comme l'affirment ses détracteurs, n'était pas un Cherokee, cela serait somme 
toute admirable et ferait de lui l'auteur de la plus grande performance de l'histoire de l'art contemporain : « a truly brilliant, half-century-long act of politically driven self-invention »(Cotter, 2017). L'idée a bien entendu été immédiatement relevée par America Meredith, une des plus ferventes accusatrices de Durham : «A last gap from his most ardent disciples is to suggest that his playing Cherokee was an elaborated art performance. » (Meredith, 2017: 89). De façon inattendue, les contempteurs et les défenseurs de l'artiste arrivent finalement au même constat : Jimmie Durham serait bien un trickster qui a « performé "son identité cherokee depuis le début de sa carrière. Mais les conclusions sont diamétralement opposées : dans un cas, il s'agit d'une fraude identitaire qui a causé un tort incommensurable à la culture cherokee puisque l'imposteur est devenu la « voix » cherokee du monde de l'art contemporain; de l'autre, il s'agit d'une admirable carrière construite sur un faux-semblant, digne héritière de Marcel Duchamp.

\section{III. Être un artiste contemporain autochtone aujourd'hui}

L'antagonisme de ces interprétations se charge d'une valeur heuristique. II fait apparaître au grand jour les incompréhensions qui existent entre un monde de l'art contemporain globalisé et des artistes autochtones engagés dans un mouvement de lutte décoloniale et de reconstruction culturelle. Nous aimerions, en guise de conclusion, nous arrêter sur cette double dimension, qui nous permettra, au-delà de la polémique autour de Jimmie Durham, de mieux faire ressortir les caractéristiques que partagent aujourd'hui les artistes contemporains autochtones.

\section{Lutte coloniale et autodétermination}

Les artistes autochtones appartiennent tous à des peuples qui ont été colonisés et qui le sont encore. La dénonciation de l'oppression et de la dépossession coloniale est présente de multiples façons chez tous ces artistes. On ne saurait faire un faux procès à Jimmie Durham sur cette question, puisqu'aussi bien dans son engagement politique que dans son art et ses écrits, la critique du colonialisme états-unien est omniprésente. Cependant, sa position contraste avec celle de la très grande majorité des artistes contemporains autochtones dans le fait que Durham semble avoir « jeté l'éponge » de la lutte anticoloniale. Sa remise en question de la légitimité du gouvernement cherokee de Tahlequah (Oklahoma), son installation définitive en Europe au début des années 90 , son refus de répondre à ses contempteurs dans la querelle actuelle laissent entendre que, pour lui, la cause de la décolonisation est perdue. Cette attitude se démarque fortement du mouvement actuel de revendication planétaire qui s'appuie sur le principe d'autodétermination des peuples autochtones, ancré dans la Déclaration des Nations Unies sur le droit des peuples autochtones, principe qui stipule que « les peuples autochtones ont le droit à l'autodétermination. En vertu de ce droit, ils déterminent librement leur statut politique et assurent librement leur développement économique, social et culturel » ([s. a.], 2007: 5). Ce droit est aujourd'hui défendu par la très grande majorité des intellectuels et décideurs autochtones, à l'instar 
du théoricien mohawk Taiaiake Alfred, qui déclare :

Qui est Autochtone? Pour avoir quelque valeur que ce soit dans le processus de rétablissement après le colonialisme, la réponse à cette question doit respecter l'intégrité des nations autochtones et de leurs traditions, et elle doit rejeter les catégories qui créent des divisions inventées par l'État. Ni la froide linéarité de la quantité de sang indien ni la faiblesse tourmentée de l'auto-identification — deux systèmes conçus et actuellement validés par l'État - ne peuvent soutenir les nations autochtones. (Alfred: 171172.)

Ce principe d'autodétermination est toutefois difficile à accepter, et encore plus à mettre en œuvre, pour les institutions du monde de l'art contemporain puisqu'il suppose de reconnaître qu'elles participent d'un système néocolonial et qu'elles acceptent de prendre les moyens nécessaires pour y remédier.

\section{Affirmation culturelle}

La deuxième dimension que met en évidence toute cette polémique est liée au mouvement d'affirmation, ou de reconstruction, culturelle des peuples autochtones. Le fait que Jimmie Durham ait explicitement rompu avec la Nation Cherokee et qu'il n'entretienne plus aucun lien avec ses membres depuis trente ans est un élément majeur de la querelle de l'été dernier. Bien entendu, Durham s'est largement expliqué sur ce divorce motivé selon lui par la collusion entre les trois tribus cherokees officielles et le pouvoir colonial. Si cette justification est, elle aussi, très bien reçue par les milieux de l'art occidentaux toujours avides des postures politiques qui dénoncent l'injustice et l'oppression, elle passe toutefois beaucoup moins bien auprès des communautés autochtones. Comment, en effet, revendiquer un statut de personne cherokee en tournant délibérément le dos à la culture, aux valeurs et au mode de vie de la communauté à laquelle on estime appartenir? C'est ce que signalaient les auteurs de la lettre de l'Indian Country Today: "Durham has no Cherokee relatives; he does not live in or spend time in Cherokee communities; he does not participate in dances and does not belong to a ceremonial ground. » (Watts, 2017.) Cet argument, dont se moquait Paul Chaat Smith dans sa conférence $\frac{10}{}$, est néanmoins central dans la présente querelle. La position de rupture que revendique Durham est d'autant plus difficile à justifier que nous vivons depuis une dizaine d'années un moment d'affirmation culturelle et artistique des Autochtones, où la posture critique, sans avoir disparu, n'est plus dominante. Aujourd'hui de plus en plus d'artistes visuels autochtones, comme Brian Jurgen et Duane Linklater (Modest Livelihood, 2012), Maria Hupfield (Bound with Georgian Bay by Peggy Miller [1974] 2016) ou Terrance Houle (Urban Indian Series, 2004), font une place à leur communauté, et même aux membres de leur famille dans leurs pratiques artistiques. L'édition 2018 de la Biennale d'art contemporain de Montréal, consacrée au thème de la sororité, est le dernier exemple de cette tendance. Même les artistes de la génération du « trickster shift » (Ryan, 1999), à laquelle appartient pourtant Durham, s'impliquent dans le sort des membres de leurs communautés, à 
l'exemple de Rebecca Belmore qui fut dès 2002 l'une des premières personnes à dénoncer le sort des femmes et des filles autochtones disparues et assassinées au Canada dans sa performance Vigil.

Ainsi, la question centrale de la polémique déclenchée parJimmie Durham. At the Center of the Worldne porte-t-elle pas tant sur les origines cherokees supposées ou réelles de l'individu, mais sur le fait de savoir si un artiste peut se présenter comme une personne autochtone alors qu'il n'entretient plus de relations avec sa communauté et sa culture depuis des décennies, et qu'il semble avoir définitivement renoncé à juguler les effets du colonialisme.

1. DURHAM, Jimmie. 1993. " An Open Letter on Recent Developpements in the American Indian Movement/International Indian Treaty Council » (1980), dans A Certain Lack of Coherence. Writings on Art and Cultural Politics . Londres : Kala Press, p. 51.

2. L'impact international de cette querelle est beaucoup plus important que celle, par exemple, qui a eu lieu en décembre 2016 autour des origines autochtones, réelles ou imaginaires, de l'écrivain canadien Joseph Boyden.

3. Nous aurons l'occasion de revenir sur ce concept plus loin.

4. Ces trois tribus, officiellement reconnues par l'État fédéral américain, ont seules le pouvoir de déterminer qui est citoyen cherokee et qui ne l'est pas. Elles ont chacune leur propre critère d'inscription : la Eastern Band of Cherokee Indians exige un minimum d'un seizième de sang Cherokee, la United Keetoowah Band of Cherokee Indians of Oklahoma un quart de sang et la Cherokee Nation requiert qu'un ancêtre soit identifié sur les Dawes Rolls établis entre 1898 et 1914.

5. L'exposition a été présentée au Hammer Museum de Los Angeles (29 janvier au 7 Mai 2017), au Walker Art Centre de Minneapolis (22 juin au 8 octobre 2017), au Whitney Museum of American Art de New York (3 novembre 2017 au 28 janvier 2018) et est présentée en ce moment au Remai Modern de Saskatoon (23 mars au 12 août 2018).

6. C'est-à-dire un ensemble de terres situées à l'ouest du Mississipi qui n'avaient pas encore été colonisées par les États-Unis. Ces limites géographiques furent fixées par The Indian Trade and Intercourse Act de 1834.

7. Les résultats de cette enquête sont détaillés sur le site de First American Art Magazine.

8. Répondre à ses accusateurs aurait signifié pour Jimmie Durham accorder de la valeur à leur critique. On se souvient toutefois qu'en avril 2011, le président Barack Obama avait fait cesser les rumeurs sur le fait qu'il n'était pas né en sol américain en publiant son acte de naissance. II n'est pas incongru de penser qu'un Jimmie Durham né en Arkansas au sein d'une famille possédant des origines cherokees aurait eu tout à gagner à produire le même type de document.

9. Le lecteur intéressé par ce débat pourra se référer utilement à deux sites qui rassemblent plusieurs documents consacrés à la polémique. Celui du Whitney Museum, qui a réuni, dans une section «Perspectives ", une série de documents dont plusieurs à " décharge " (entre autres, les réactions d'Anne Ellegood et de Paul Chaat Smith). Celui du First American Art Magazine, très clairement à charge, qui offre des éléments généalogiques sur la famille de Jimmie Durham.

10. "Now, l'll admit l'm not a very good citizen of the Comanche Nation. [...] If you applied the standards some advocate, things like actively participating in cultural activities, learning songs and dances and so forth, I would be disenrolled in a heartbeat 》 (Smith, 2017).

\section{Bibliographie}

[s. a.]. 2007. Déclaration des Nations Unies sur le droit des peuples autochtones. New York : Organisation des Nations Unies, pdf, 16 p. <http://www.un.org/esa/socdev/unpfii/documents/DRIPS fr.pdf>.

AlfRed, Taiaiake. 2014. Paix, pouvoir et droiture. Un manifeste autochtone, 2e édition, traduit par Caroline Pageau. Wendake : Éditions Hannenorak, 303 p.

Cotter, Holland. 2017. «Coming Face to Face With Jimmie Durham». The New York Times, 3 novembre 
2017, p. C15. <https://www.nytimes.com/2017/11/02/arts/design/jimmie-durham-review-whitney-museumcherokee.html>.

Durham, Jimmie. 1993. A Certain Lack of Coherence. Writings on Art and Cultural Politics Londres : Kala Press, $255 \mathrm{p}$.

Durham, Jimmie, Nancy Marie Mitchell et Lucy LiPPARd. 2017 [1993]. «Letters. Identities Clarified? », correspondances. Art in America, 15 août. <https://www.artinamericamagazine.com/news-

features/magazines/letters-identities-clarified/>. Consultée le 15 mai 2018.

DurHam, Jimmie. 2014. Jimmie Durham, Waiting to Be Interrupted. Selected Writings 1993-2012

Milan/Anvers : Mousse Publishing, 368 p.

ELDRED, Sheila M. 2017. «Walker Art Center’s Reckoning With 'Scaffold' Isn’t Over Yet». The New York Times, 14 septembre 2017, p. C1. 丸ttps://www.nytimes.com/2017/09/13/arts/design/walker-art-centerscaffold.html>.

a

Ellegood, Anne. 2017. Jimmie Durham. At the Center of the World, catalogue d'exposition. Munich : Prestel, $320 \mathrm{p}$.

b

ElleGood, Anne. 2017. «Curator Anne Ellegood on Understanding the Complexities of Jimmie Durham's Native Identity ». Artnet News, 2 août. «https://news.artnet.com/opinion/anne-ellegood-jimmie-durham1033907>.

FINKEL, Jori. 2017. «The Artist Jimmie Durham. A Long Time Gone, but Welcomed Back». The New York Times, 12 mars 2017, p. AR20. łttps://www.nytimes.com/2017/03/10/arts/design/the-artist-jimmie-durhama-long-time-gone-but-welcomed-back.html>.

Hessel, Ingo. 2010. «"I Am an Artist". Inuit Art Transcends Ethnicity », dans Gerald McMaster (dir.), Inuit modern : the Samuel and Esther Sarick Collection. Toronto; Vancouver : Art Gallery of Ontario; Douglas \& Mclntyre, p. 187-192.

LALONDE, Christine. 2013. «Introduction. Au carrefour de l'indigénéité, de la mondialisation et de l'art contemporain », dans Christine Lalonde, Greg A Hill et Candice Hopkins (dir.), Sakahàn. Art indigène international. Ottawa : Musée des beaux-arts du Canada, p. 14-20.

LIPPARD, Lucy. 1993. « Jimmie Durham. Postmodernist "Savage” ». Art in America, vol. 81, no 2, p. 62-68.

Meredith, America. 2017. «A Chapter Closed? ». First American Art Magazine, no 16, p. 88-89.

Papastergiadis, Nikos et Laura Turney. 1996. On Becoming Authentic. Interview with Jimmie Durham Cambridge (UK) : Ricky Pear Press, $53 \mathrm{p}$.

Russel, Steven. 2008. «When Does Ethnic Fraud Matter?». Indian Country Media Networks. $<$ https://indiancountrymedianetwork.com/news/russell-when-does-ethnic-fraud-matter-2/>>. 
Ryan, Allan J. 1999. The Trickster Shift Humour and Irony in Contemporary Native Art Vancouver; Seattle : University of British Columbia Press; University of Washington Press, $303 \mathrm{p}$.

SmITH, Paul Chaat. 2009. Everything You Know About Indians is Wrong. Minneapolis : University of Minnesota Press, $194 \mathrm{p}$.

SмIтH, Paul Chaat. 2017. «On Jimmie Durham, Native Identity, and Americans. The Forthcoming Smithsonian Exhibition ». Walker Magazine. <https://walkerart.org/magazine/paul-chaat-smith-jimmiedurham-americans-nmai-smithsonian>.

TILOVE, Jonathan. 1993. «Under Arts and Crafts Act. Only Card-carrying Indians Can Create 'Indian Art'». Newhouse News Service, 16 mai. 丸ttps://jonathantilove.com/indian-ar/>.

TuRney, Laura. 1999. «Ceci n'est pas Jimmie Durham ». Critique of Anthropology, vol. 19, no 4, p. 423-442. Watts, Cara Cowan, Meredith America, Kade Twist, Lynne Harlan et al.. 2017. «Dear Unsuspecting Public, Jimmie Durham Is a Trickster ». Indian Country Today, 26 juin.

$<$ https://indiancountrymedianetwork.com/news/opinions/dear-unsuspecting-public-jimmie-durhamtrickster/>.

WeinberG, Adam D. 2018. «Director's Statement. "Jimmie Durham. At the Center of the World”". Whitney Museum of American Art. New York: Whitney Museum of American Art. $<$ https://whitney.org/DirectorsStatementJimmieDurham>.

WoodWARD, Grace Steel. 1963. The Cherokees. Norman : University of Oklahoma Press, 359 p. 